\title{
Tribological testing of self-mated nanocrystalline diamond coatings on $\mathrm{Si}_{3} \mathrm{~N}_{4}$ ceramics
}

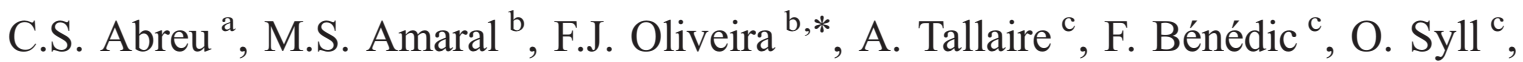 \\ G. Cicala ${ }^{\mathrm{d}}$, J.R. Gomes ${ }^{\mathrm{e}}$, R.F. Silva ${ }^{\mathrm{b}}$ \\ ${ }^{a}$ Physics Department, Porto Superior Engineering Institute, ISEP, 4200-072 Porto, Portugal \\ ${ }^{\mathrm{b}}$ Ceramics and Glass Eng. Department, CICECO, Univ. of Aveiro, 3810-193 Aveiro, Portugal \\ ${ }^{\mathrm{c}}$ LIMHP, UPR 1311 CNRS, Université Paris 13, 93430 Villetaneuse, France \\ d IMIP-CNR Sezione di Bari, University of Bari, 70126 Bari, Italy \\ e Mechanical Eng. Department, CIICS, University of Minho, 4800-058 Guimarães, Portugal
}

Available online 4 January 2006

\begin{abstract}
Due to their much lower surface roughness compared to that of microcrystalline diamond, nanocrystalline diamond (NCD) films are promising candidates for tribological applications, in particular when deposited on hard ceramic materials such as silicon nitride $\left(\mathrm{Si}_{3} \mathrm{~N}_{4}\right)$. In the present work, microwave plasma-assisted chemical vapour deposition of NCD is achieved using $\mathrm{Ar} / \mathrm{H}_{2} / \mathrm{CH}_{4}$ gas mixtures on plates and ball-shaped $\mathrm{Si}_{3} \mathrm{~N}_{4}$ specimens either by a conventional continuous mode or by a recently developed pulsed regime. The microstructure, morphology, topography and purity of the deposited films show typical NCD features for the two kinds of substrate shapes. Besides, tribological characterization of the NCD/ $\mathrm{Si}_{3} \mathrm{~N}_{4}$ samples is carried out using self-mated pairs without lubrication in order to assess their friction and wear response. Worn surfaces were studied by SEM and AFM topography measurements in order to identify the prevalent wear mechanisms. Friction values reached a steady-state minimum of approximately 0.02 following a short running-in period where the main feature is a sharp peak which attained a maximum around 0.44. Up to the critical load of $35 \mathrm{~N}$, corresponding to film delamination, the equilibrium friction values are similar, irrespective of the applied load. The calculated wear coefficient values denoted a very mild regime $\left(K \sim 1 \times 10^{-8} \mathrm{~mm}^{3} \mathrm{~N}^{-1} \mathrm{~m}^{-1}\right)$ for the self-mated NCD coatings. The predominant wear mechanism was identified as self-polishing by micro-abrasion.
\end{abstract}

(C) 2005 Elsevier B.V. All rights reserved.

Keywords: Nanocrystalline diamond; Silicon nitride; Tribological characterization

\section{Introduction}

In the past few years, an increasing interest has been focused on the synthesis of nanocrystalline diamond films (NCD) due to their extreme hardness and their nanometre-scale grain size which ensures a very low surface roughness [1,2]. However, the mechanical and tribological applications of such coatings are mainly limited by the weak properties of the substrates used since depositions are usually performed on silicon. Recently the possibility to use silicon nitride $\left(\mathrm{Si}_{3} \mathrm{~N}_{4}\right)$ ceramics as substrates has been pointed out $[3,4]$. Indeed $\mathrm{Si}_{3} \mathrm{~N}_{4}$ is a tough and hard material that ensures load-bearing capability for mechanical purposes and an adequate affinity and adhesion with diamond.

\footnotetext{
* Corresponding author. Tel.: +351 234370 266; fax: +351 234425300 .

E-mail address: filipe@cv.ua.pt (F.J. Oliveira).
}

NCD films deposited on silicon nitride substrates could find applications in numerous fields such as biomaterials, mechanical face seals, bearings or cutting tools. Diamond films have a very low friction and high wear resistance under a wide range of sliding contact conditions [5] whose performance may be improved if ultra-smooth NCD films are used. Values of the friction coefficient in the range of $0.03-0.06$ were obtained in self-mated fine grain microcrystalline diamond coatings [6]. Also, high values were measured in the early phases of wear tests of microcrystalline diamond films $[7,8]$. It is then of great interest to further assessing the tribological properties of NCDcoated $\mathrm{Si}_{3} \mathrm{~N}_{4}$ substrates in what matters their friction and wear behaviour.

High quality homogeneous NCD films can be deposited on $\mathrm{Si}_{3} \mathrm{~N}_{4}$ substrates with typical growth rates of a few micrometers per hour using microwave plasma assisted chemical vapour 
deposition (MPACVD) process working in a conventional continuous mode [4]. Controlling the deposition temperature is, however, quite difficult when depositing on complex-shape substrates since the dielectric sample may deeply penetrate the discharge which leads to a pronounced edge effect and to a subsequent graphitisation of the film [9]. This phenomenon is particularly important for $\mathrm{Si}_{3} \mathrm{~N}_{4}$ material due to its low thermal conductivity, but can be successfully overcome by the use of an appropriate pulsed microwave discharge [9].

In this work, the tribological properties of $\mathrm{Si}_{3} \mathrm{~N}_{4}$ ceramic substrates coated with a NCD thin layer are investigated. The diamond depositions were performed under $\mathrm{Ar} / \mathrm{H}_{2} / \mathrm{CH}_{4}$ microwave discharges both on silicon nitride plates and on ballshaped substrates in continuous wave $(\mathrm{CW})$ and pulsed wave (PW) mode, respectively. Tribological characterization of such NCD films is achieved using self-mated pairs (ball and plate) without lubrication in order to assess their friction coefficient values and wear behaviour when subjected to such extreme surface contacts.

\section{Experimental details}

Dense $\mathrm{Si}_{3} \mathrm{~N}_{4}$ plates $\left(10 \times 10 \times 3 \mathrm{~mm}^{3}\right)$ and balls $(5 \mathrm{~mm}$ diameter) were used as substrates for diamond deposition. The mirror polished plates were produced by pressureless sintering at $1750{ }^{\circ} \mathrm{C} / 2 \mathrm{~h}$ under nitrogen atmosphere, followed by cutting, grinding, lapping and finally polishing with colloidal silica. The balls, commercially available (Kema Nord), were polished down to $1 \mu \mathrm{m}$ with diamond slurry before scratching for NCD growth. NCD deposition was carried out using previously optimised growth conditions in a bell-jar type reactor $[4,9]$. Prior to the plasma treatment, all the samples (plates and balls) were submitted to an appropriate ultrasonic pre-treatment in a $40 \mu \mathrm{m}$ grain size diamond powder suspension in order to enhance diamond nucleation on the substrate surface [10]. Two ceramic plates, referred as $\mathrm{P} 1$ and $\mathrm{P} 2$, were successively recovered by a thin $\mathrm{NCD}$ layer $(\sim 12 \mu \mathrm{m})$ using the conventional CW mode. The feed gas was an $\mathrm{Ar} / \mathrm{H}_{2} / \mathrm{CH}_{4}$ mixture (96\%:3\%:1\%) with a total flow rate of $250 \mathrm{sccm}$. The pressure in the chamber was set to 200 mbar while the injected microwave power was maintained constant at $600 \mathrm{~W}$. The surface temperature monitored with a bichromatic infrared pyrometer was controlled using an additional heating system located in the substrate holder and set at 925 and $825^{\circ} \mathrm{C}$ for samples $\mathrm{P} 1$ and $\mathrm{P} 2$, respectively. In order to avoid the edge effect induced by the shape of the substrates, the two $\mathrm{Si}_{3} \mathrm{~N}_{4}$ balls, labelled B1 and B2, were coated under PW discharges. The gas mixture and flow rate were the same than those described above, but in this case the input power was modulated with a pulse repetition rate of $1000 \mathrm{~Hz}$ and a duty cycle of $50 \%$ for a maximum input microwave power of $800 \mathrm{~W}$. The balls were carefully placed in a circular hole made in the molybdenum substrate holder, the depth of which was approximately half their diameter. The surface temperature was estimated for both samples at $925^{\circ} \mathrm{C}$. The growth duration imposed for each sample was $3 \mathrm{~h}$ to give a final thicknesses of approximately $12 \mu \mathrm{m}$ for the flat substrates.
The microstructure, morphology, topography and purity of NCD films deposited on $\mathrm{Si}_{3} \mathrm{~N}_{4}$ ceramic plates and ball-shaped substrates in the conditions described above were discussed in previous papers $[4,9]$. The main features are briefly summarized in the next section where observations by scanning electron microscopy (SEM) (Hitachi S4100), atomic force microscopy (AFM) (Digital Instrument Multimode IIIa) and micro-Raman spectroscopy (high-resolution confocal spectrometer JobinYvon HR800) are also reported.

The tribological characterization of the NCD films was achieved using a ball-on-flat reciprocating tribometer (PLINT TE67/R). Unlubricated self-mated tests were performed in ambient atmosphere (RH of 50-60\%) at room temperature, with constant stroke $(6 \mathrm{~mm})$ and frequency $(1 \mathrm{~Hz})$. The NCD-coated balls (upper specimen) were mounted in the sample holder arm and made to contact with a defined applied load onto the counter surface. The oscillatory relative motion between the opposing surfaces was obtained by clamping NCD-coated square plate counterbodies (lower specimen) on a reciprocating table. The applied normal load varied in the range of 10 to $40 \mathrm{~N}$. Considering a Hertzian elastic contact for a sphere pressed by a determined load into a flat specimen, the applied loads used in this study produced initial peak pressures under the ball in the range of 5-8 $\mathrm{GPa}[11,12]$. All tribological experiments were conducted over a sliding distance $(x)$ of $43 \mathrm{~m}$, for the normal tests, and $690 \mathrm{~m}$ for the endurance tests.

Friction scans were completed during the sliding tests by means of a load cell, previously calibrated by applying known dead-weights in the range of the measured loads. The wear coefficient $(k)$ of the NCD-coated balls was assessed from the volumetric wear $(V)$ of this triboelement determined from SEM observations of the near circular wear scars produced in the contact zone [8].

After completion of the sliding tests, the worn surfaces were examined by SEM and AFM (Digital Instrument NanoScope IIIa) with the purpose of ascertain the morphological and topographical characteristics of the films subjected to damage stress, and correlate the results with the friction and wear mechanisms of NCD films in ambient atmosphere.

\section{Results and discussion}

\subsection{Characterization of the NCD films}

All the NCD films synthesized either under CW or PW discharges exhibit smooth granular surfaces where no facetted crystallites can be noticed. Typical SEM and AFM images obtained on sample P2 are presented in Fig. 1 as an illustration. The SEM micrograph (Fig. 1a) emphasises the full recovering and uniformity of the NCD films, whereas the AFM image (Fig. 1b) points out their surface smoothness, with typical surface roughness values $\left(R_{a}\right)$ in the range between 20 and $40 \mathrm{~nm}$. For the ball-shaped samples B1 and B2, the deposit consisted in a hemispherical NCD film recovering homogeneously the upper part of the balls since only a portion of the substrates was exposed to the plasma due to their position on the substrate holder. 
(a)
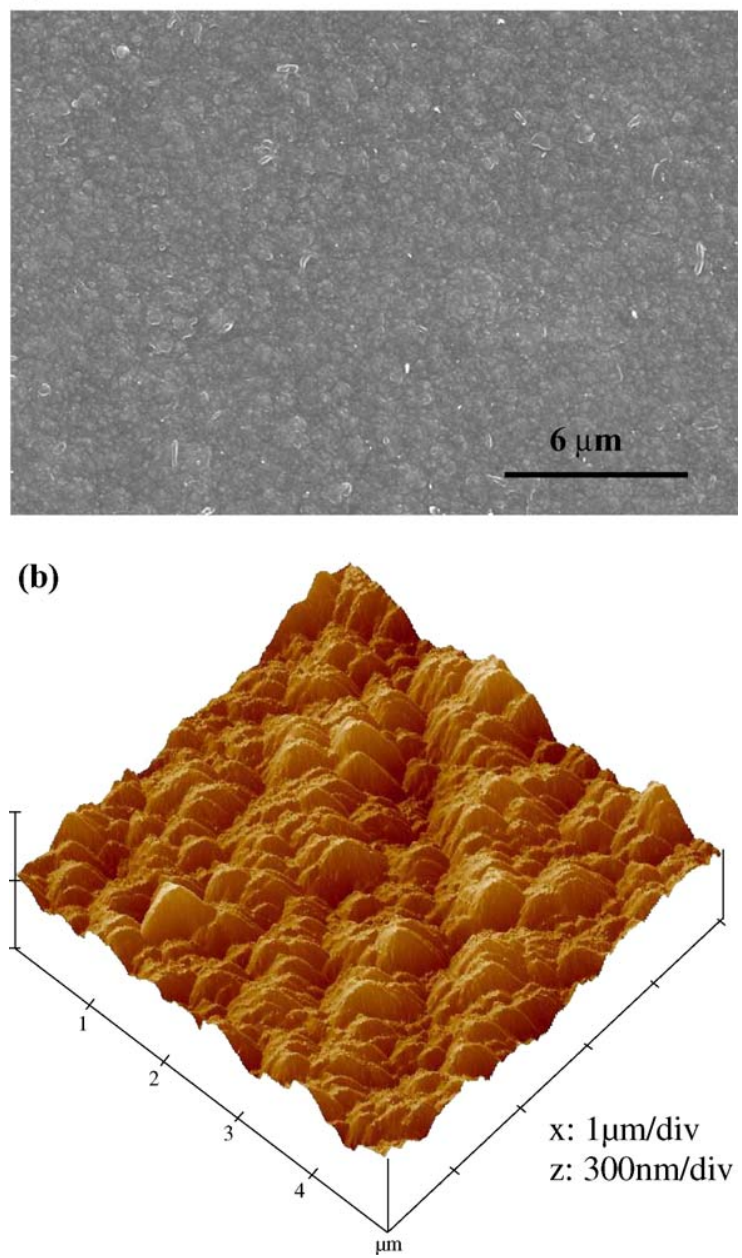

Fig. 1. Typical morphology and topography of the deposited NCD films as observed for sample P2 by: (a) scanning electron microscopy; (b) atomic force microscopy on a $5 \times 5 \mu \mathrm{m}^{2}$ surface.

Raman spectroscopy of the samples was performed using the $488 \mathrm{~nm}$ line of an $\mathrm{Ar}^{+}$laser as the excitation wavelength (Fig. 2). Typical NCD features are observed for both plates and ball-shaped substrates, in particular the trans-polyacetylene bands at 1150 and $1480 \mathrm{~cm}^{-1}$, the graphite D and $\mathrm{G}$ bands around 1350 and $1580 \mathrm{~cm}^{-1}$, respectively, and a weak broad diamond peak at $1332 \mathrm{~cm}^{-1}[13,14]$. Note that, in the spectrum of sample P1, the trans-polyacetylene bands and the diamond peak have a weaker intensity with regard to sample P2. This may be due to the higher deposition temperature imposed for sample P1 since an increase of the surface temperature was shown to provoke a transition towards more graphite-like NCD layers [15]. The XRD analysis done in the NCD films deposited on $\mathrm{Si}_{3} \mathrm{~N}_{4}$ plates give evidence a good crystallinity through the observation of the characteristic $<111>$ and $<311>$ diffraction peaks. The grain size was estimated around $12 \mathrm{~nm}$ from the broadening of the $<111>$ peak [9]. By measuring the thickness of the films on cross-section SEM images, the growth rate of the NCD layers on the ceramic plates was estimated to be about $4 \mu \mathrm{m} \mathrm{h}^{-1}$.

\subsection{Tribological behaviour}

A first set of ball-on-flat reciprocal tests was carried out to determine the maximum critical load the NCD films could withstand. The NCD coatings sustained stable operation, without film breakage, under tribological action for applied loads up to $35 \mathrm{~N}$ that corresponds to a maximum contact pressure of $\sim 6.8 \mathrm{GPa}$.

Fig. 3 shows typical friction scans of self-mated NCD coatings on $\mathrm{Si}_{3} \mathrm{~N}_{4}$ ceramics as a function of the sliding distance. The represented curves reveal a running-in effect with a pronounced peak as its main feature, followed by a stationary phase with extremely low friction coefficients. The initial friction coefficient of examined NCD coatings is in the range from 0.13 to 0.44 and denoted a significant dependence on the starting surface roughness of as-deposited films. This behaviour exists in spite of the nano-scale nature of the surface roughness of the NCD thin films, and has been referred in some studies involving fine-grained $(20-100 \mathrm{~nm})$ polycrystalline self-mated CVD diamond coatings [16]. This same feature was observed for larger grain sized MPACVD diamond films $(1.8-4.6 \mu \mathrm{m})$ in experiments similar to those of the present work [8]. This suggests that the initial friction regime is predominantly caused

(a)

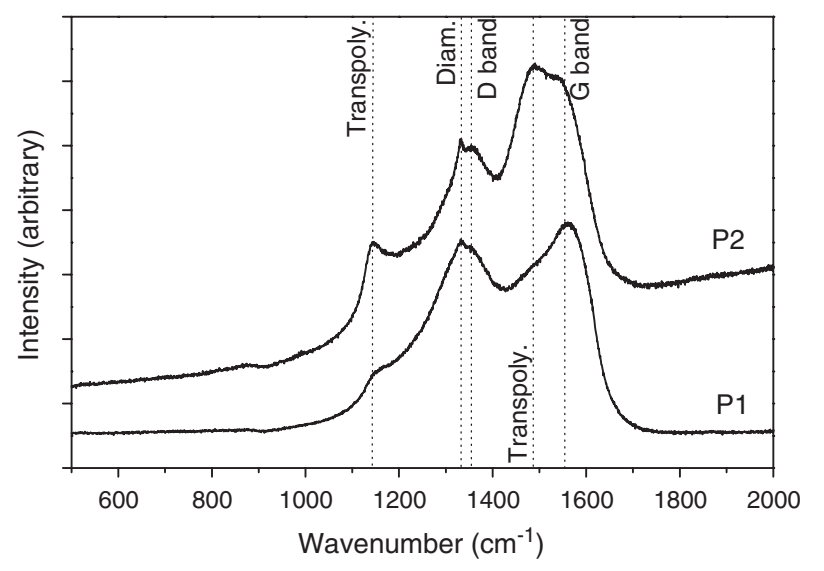

(b)

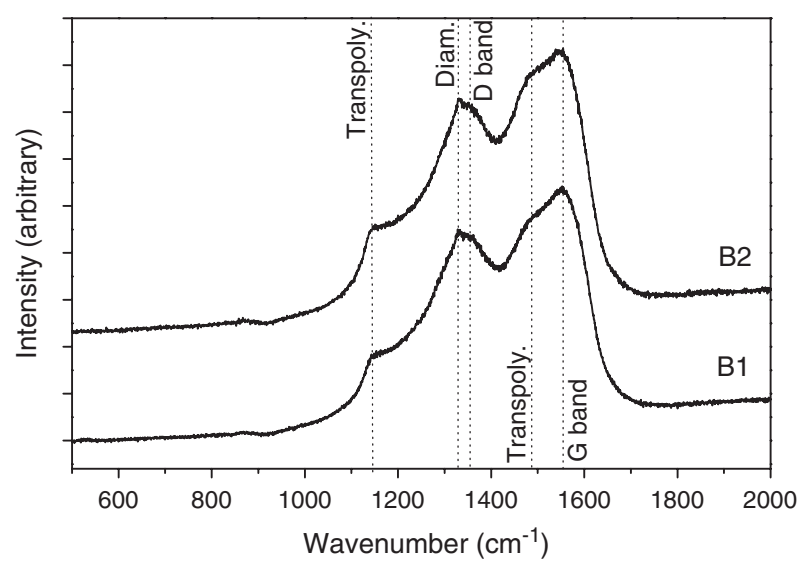

Fig. 2. Raman spectra of the NCD films deposited on the silicon nitride substrates: (a) P1 and P2 plates; (b) B1 and B2 balls. The excitation line was 488 $\mathrm{nm}$ and the main bands are indexed on the spectra. 


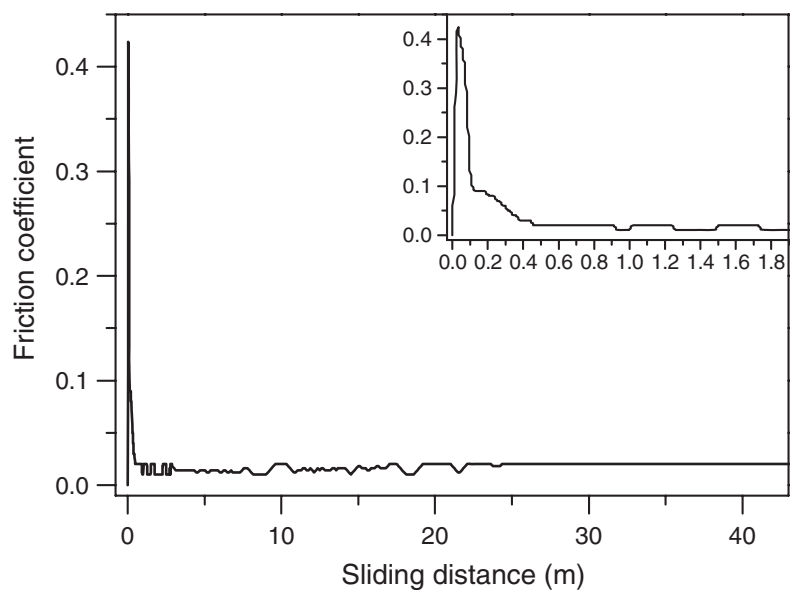

Fig. 3. Representative evolution of the friction curve, for unlubricated self-mated NCD films in ambient atmosphere, as a function of the sliding distance on a sample tested for $x=43 \mathrm{~m}$ under $W=30 \mathrm{~N}$.

by a strong interaction between surface asperities, i.e. by a mechanism of fragmentation and deformation of contacting asperities. This process can be observed in the SEM micrograph of Fig. 4a, where a change from the nominal NCD topography (Fig. 1a) to more flattened surfaces took place. In this case, there is not a large degree of polishing due to the low load (10 N) and short sliding distance $(43 \mathrm{~m})$. For longer distances, the surface

\section{(a)}

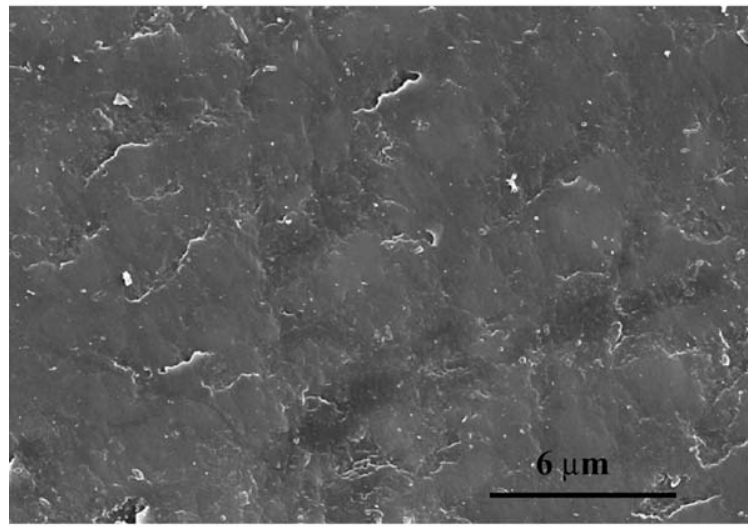

(b)

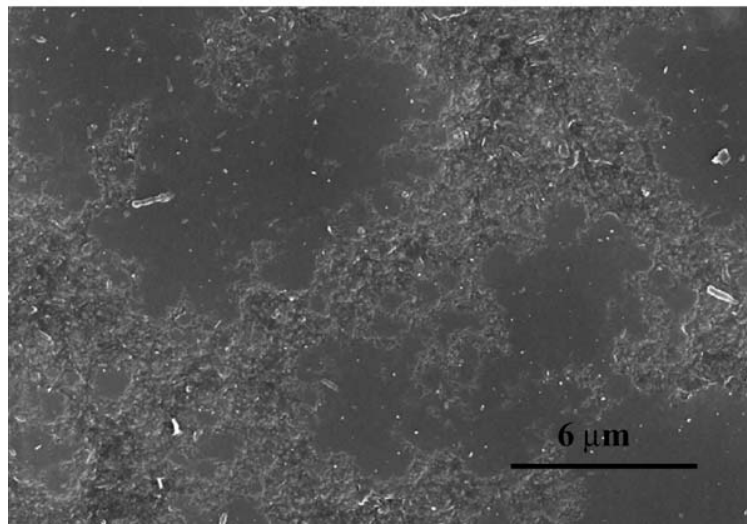

Fig. 4. SEM micrographs of NCD films revealing the smooth morphology of worn disc specimens: (a) $x=43 \mathrm{~m}, W=10 \mathrm{~N}$ at the centre of the wear track; (b) $x=690 \mathrm{~m}, W=10 \mathrm{~N}$ near the border of the track, showing ultra-smooth plateaus. becomes fully flattened, revealing extensive polishing. The micrograph in Fig. 4b was taken from the border of the wear track of a test conducted for $690 \mathrm{~m}$ under $10 \mathrm{~N}$. In the centre of the track, the NCD film is fully flat (lack of topographic contrast under SEM), with surface roughness values of about $2 \mathrm{~nm}$ (rms) determined by AFM, while laterally it still contains unpolished zones.

As sliding continues, an equilibrium track is reached with the nano-asperities worn down. The relevance of the interlocking mechanism gradually looses its importance in the overall friction phenomena thus giving very low steady-state friction values $(0.02-0.04)$. This idea is reinforced by the well known fact that the adhesive component of friction is highly reduced during the sliding of smooth passivated diamond surfaces in ambient atmosphere, following saturation of the dangling carbon bonds by adsorbed species [17]. Another feature observed in this study was that the steady-state friction values remained the same, irrespective of the starting surface roughness of as-deposited NCD films and applied load. Miyoshi [16] reports a similar behaviour in terms of the starting surface roughness influence in the final equilibrium friction of selfmated fine-grained polycrystalline diamond. In addition, as can be seen from the inset of the friction curve of Fig. 3, the levelling of the friction coefficient is readily achieved after a few decimeters of sliding distance, thus indicating a short running-in regime for homologous pairs of NCD in open air.

The AFM micrographs shown in Fig. 5 reveal the differences between the centre and sides of wear tracks produced at short
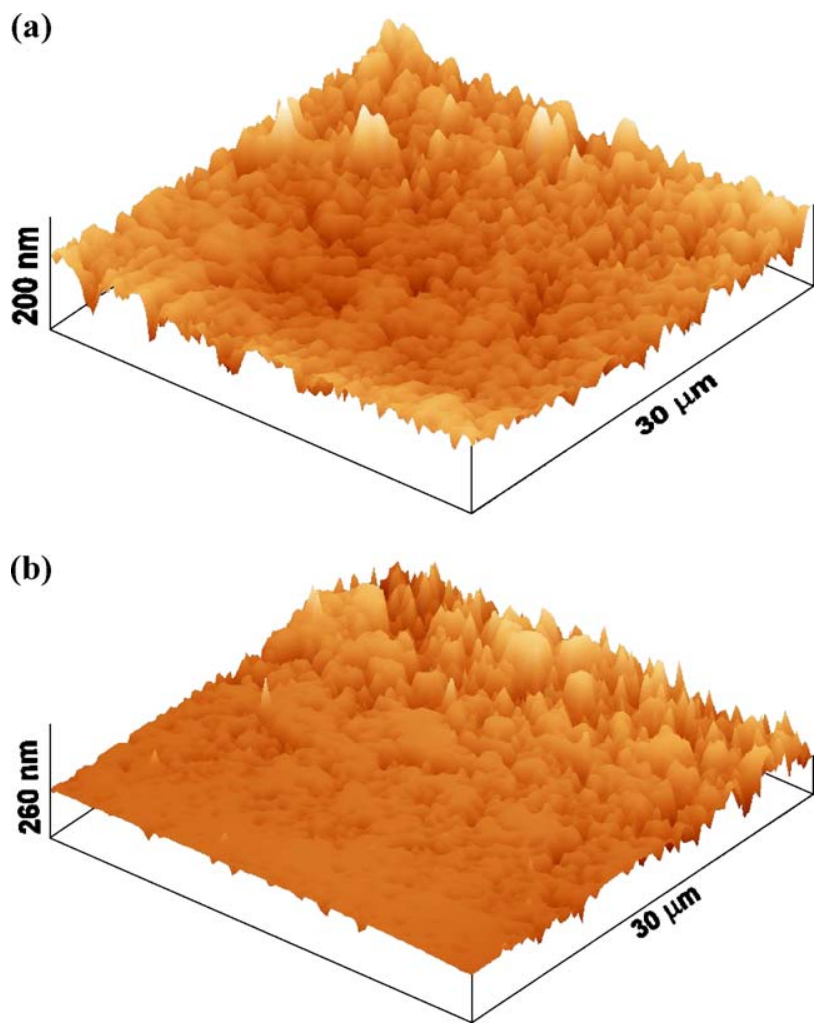

Fig. 5. AFM scans of worn NCD coatings taken from the borders of wear tracks on disc specimens using tapping mode: (a) $x=43 \mathrm{~m}, W=35 \mathrm{~N}$; (b) $x=690 \mathrm{~m}$, $W=30 \mathrm{~N}$. 
(Fig. 5a) and long (Fig. 5b) running distances under $35 \mathrm{~N}$ and 30 $\mathrm{N}$ loads, respectively. Even for the $43 \mathrm{~m}$ experiment, the centre of the track is already very smooth ( $\mathrm{rms} \sim 7 \mathrm{~nm}$ ), unlike the case of the $10 \mathrm{~N}$ test (Fig. 4a). For longer distances, the surfaces become even smoother as the AFM micrograph of Fig. 5b clearly evidences. Surface roughness values in the range of $\sim 2$ $\mathrm{nm}$ (rms) are achieved. The observed morphology and this extremely flattened topography are characteristic of a selfpolishing wear mechanism by micro-abrasion.

The wear coefficient values of balls sliding against NCD plate specimens varied in the range of $1.0-1.4 \times 10^{-8} \mathrm{~mm}^{3} \mathrm{~N}^{-1}$ $\mathrm{m}^{-1}$, indicating a very mild wear regime in ambient atmosphere for the long duration tests $(690 \mathrm{~m})$. It is worth noting that the wear of the NCD plate specimens was not measurable by standard techniques (weight loss and conventional profilometry) although the NCD film becomes smoothened as described above (Figs. 4b and 5b).

Although the deposited NCD diamond films contain some non-diamond phases (Fig. 2), there is no apparent effect of their presence on the friction coefficients or wear resistance. The main differences relatively to wear tests conducted with selfmated microcrystalline diamond are the lower starting friction coefficient peaks and shorter running-in periods. These can be accounted for entirely by the much smaller starting surface roughness of NCD films relatively to polycrystalline diamond ones. Thus, the role of graphitic phases on the wear behaviour of these NCD-coated $\mathrm{Si}_{3} \mathrm{~N}_{4}$ ceramics is yet to be fully understood.

\section{Conclusions}

NCD films were successfully deposited by MPACVD method from $\mathrm{Ar} / \mathrm{H}_{2} / \mathrm{CH}_{4}$ gas mixtures on $\mathrm{Si}_{3} \mathrm{~N}_{4}$ ceramic plates and balls, using CW and PW modes, respectively.

Ball-on-flat tribological experiments allowed the estimation of a critical load of $35 \mathrm{~N}(\sim 6.8 \mathrm{GPa})$ for film delamination.

Friction curves show a pronounced friction peak attributed to the mechanical interlocking of surface diamond asperities, quickly followed by very low steady-state friction coefficients (0.02-0.04) caused by the decrease of surface roughness through extensive polishing mechanism. Final surface roughness reached values of the order of $2 \mathrm{~nm}(\mathrm{rms})$ after long running distances $(690 \mathrm{~m})$. Such low wear damage of the coated plates hindered a weight loss sufficient enough to be measured by usual techniques. In the balls, the calculated wear coefficient is typical of a very mild wear regime, in the range of $1.0-1.4 \times 10^{-8} \mathrm{~mm}^{3} \mathrm{~N}^{-1} \mathrm{~m}^{-1}$.
The very low friction, high wear resistance and enhanced adhesion of the NCD coatings deposited on $\mathrm{Si}_{3} \mathrm{~N}_{4}$ substrates make this tribosystem an excellent choice for dry tribological applications involving high contact stresses, such as cutting tools or mechanical seals.

\section{Acknowledgements}

C.S. Abreu and M. Amaral acknowledge PRODEP III funds and FCT for the grant SFRH/BD/9272/2002, respectively, in support of their $\mathrm{PhD}$ works. FCT is acknowledged for funding this work under project POCTI/CTM/45423/2002. This work was also supported by the CNRS/GRICES project no. 14153 and a bilateral agreement between CNR and CNRS.

\section{References}

[1] J. Lee, B. Hong, R. Messier, R.W. Collins, Appl. Phys. Lett. 69 (1996) 1716.

[2] D.M. Bhusari, J.R. Yang, T.Y. Wang, K.H. Chen, S.T. Lin, L.C. Chen, J. Mater. Res. 3 (1998) 1769.

[3] M. Amaral, F.J. Oliveira, M. Belmonte, A.J.S. Fernandes, F.M. Costa, R.F. Silva, Diamond Relat. Mater. 13 (2004) 643.

[4] M. Amaral, F. Mohasseb, F.J. Oliveira, F. Bénédic, R.F. Silva, A. Gicquel, Thin Solid Films 482 (2005) 232.

[5] A. Erdemir, G.R. Fenske, A.R. Krauss, D.M. Gruen, T. McCauley, R.T. Csencsits, Surf. Coat. Technol. 120-121 (1999) 565.

[6] L. Vandenbulcke, M.I. De Barros, Surf. Coat. Technol. 146-147 (2001) 417.

[7] P. Hollman, O. Wänstrand, S. Hogmark, Diamond Relat. Mater. 7 (1998) 1471.

[8] C.S. Abreu, F.J. Oliveira, M. Belmonte, A.J.S. Fernandes, R.F. Silva, J.R. Gomes, Wear 259 (2005) 771.

[9] P. Bruno, F. Bénédic, A. Tallaire, F. Silva, F.J. Oliveira, M. Amaral, A.J.S. Fernandes, G. Cicala, R.F. Silva, Diamond Relat. Mater. 14 (2005) 432.

[10] G. Cicala, P. Bruno, F. Bénédic, F. Silva, K. Hassouni, G.S. Senesi, Diamond Relat. Mater. 14 (2005) 421.

[11] P.D. Warren, J. Eur. Ceram. Soc. 15 (1995) 385.

[12] A.R. Krauss, O. Auciello, D.M. Gruen, A. Jayatissa, A. Sumant, J. Tucek, D. Mancini, N. Moldovan, A. Erdemir, D. Ersoy, M.N. Gardos, H.G. Busmann, E.M. Meyer, M.Q. Ding, Diamond Relat. Mater. 10 (2001) 1952.

[13] A.C. Ferrari, J. Robertson, Phys. Rev., B 63 (2001) 121405(R).

[14] R. Pfeiffer, H. Kuzmany, P. Knoll, S. Bokova, N. Salk, B. Günther, Diamond Relat. Mater. 12 (2003) 268.

[15] P. Bruno, F. Bénédic, F. Mohasseb, F. Silva, K. Hassouni, Thin Solid Films 482 (2005) 50.

[16] K. Miyoshi, Mater. Sci. Eng., A 209 (1996) 38.

[17] S.E. Grillo, J.E. Field, Wear 254 (2003) 945. 